\title{
Risk stratification and surgery for anomalous aortic origin of a coronary artery: Onward through the fog
}

\author{
Charles D. Fraser, Jr, MD, ${ }^{a}$ and Luis E. Martínez-Bravo, MD ${ }^{\mathrm{a}, \mathrm{b}}$
}

Anomalous aortic origin of a coronary artery (AAOCA) represents a spectrum of anatomic variations of coronary ostial origin and course with uncertain clinical implications. AAOCA is typically defined as origin of a main coronary ostium from the incorrect aortic sinus; thus there is AAOCA of the left main coronary ostium from the right aortic sinus (AAOLCA) and conversely AAOCA of the right coronary ostium from the left aortic sinus (AAORCA). The term AAOCA has also been used to include high origin of a coronary ostium at or above the aortic sinotubular junction, a single coronary ostium (either from the right or left aortic sinus), atresia of a coronary ostium, or an intraseptal course of the left main or left anterior descending coronary artery. ${ }^{1}$

AAOCA has become a lightning rod topic in congenital heart disease. The rare, but very troubling, association of AAOCA (most frequently, but not exclusively AAOLCA) with myocardial ischemia and, in some patients, unheralded sudden cardiac death (SCD) has garnered much attention. AAOCA is believed to be the second most common cause of SCD in otherwise healthy people. ${ }^{2}$ Longitudinal, objective data about the important subtleties of anatomic variants of AAOCA and their corresponding association with pathophysiology are largely lacking. The current state of understanding, or lack thereof, of the relative risk of AAOCA has been historically inferred from autopsy series and small, single-institution series of patients presenting with either symptomatology (rare) or after an incidental diagnosis of AAOCA (more frequent). ${ }^{3}$ These quite obviously very skewed patient cohorts are not believed to be representative of the scope of the anomaly, and they certainly cannot provide a complete understanding of the issues surrounding AAOCA.

Understandably, the very tragic reality of SCD in a young person with unsuspected AAOCA leads to impassioned attention, often in the lay press. Responses from the public

From the ${ }^{\text {a }}$ Texas Center for Pediatric and Congenital Heart Disease, University of Texas Dell Medical School, Dell Children's Medical Center, Austin, Tex; and ${ }^{\text {b Tec- }}$ nologico de Monterrey, Escuela de Medicina y Ciencias de la Salud, Monterrey, Mexico.

Received for publication June 21, 2019; revisions received June 21, 2019; accepted for publication June 26, 2019; available ahead of print March 9, 2021.

Address for reprints: Charles D. Fraser, Jr, MD, Texas Center for Pediatric and Congenital Heart Disease, University of Texas Dell Medical School, Dell Children's Medical Center, 4900 Mueller Blvd, Suite 3S.008, Austin, TX 78723 (E-mail: charles.fraser@austin.utexas.edu).

J Thorac Cardiovasc Surg 2021;161:1584-6

$0022-5223 / \$ 36.00$

Copyright $\Subset 2021$ Published by Elsevier Inc. on behalf of The American Association for Thoracic Surgery

https://doi.org/10.1016/j.jtcvs.2019.06.131

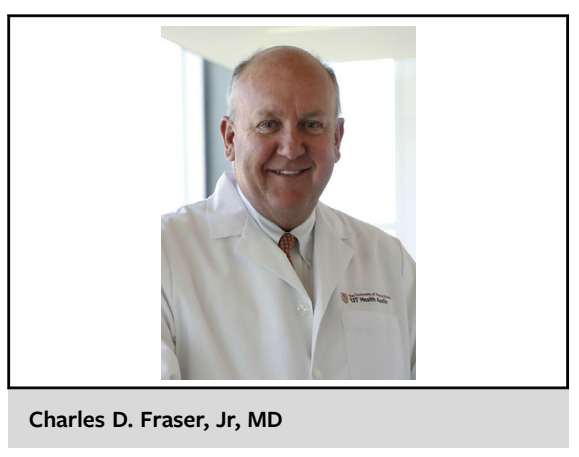

and medical communities have ranged from local institutional registries to mass screening programs in schools and athletic organizations. ${ }^{4}$ Concerned parents may seek screening echocardiograms just to make sure their children are "okay" for competitive sports or other strenuous activities. In these situations, symptom-free people may be incidentally found to have an AAOCA, which then leads to a cascade of events and emotions. To be certain, the anatomic diagnosis alone may translate into a disabling psychologic situation for a patient and their family. Uncertainty about the relevance of the anatomic findings, particularly in the absence of documented myocardial ischemia during provocative testing, can devolve into a miserable conundrum for practitioners and an overwhelming worry burden for patients and families.

The decision to offer a theoretically corrective operation, designed to reduce the risk of ischemia or SCD in association with AAOCA, is easy for patients with a history of SCD or clear evidence of ischemia - these patients must undergo surgery. ${ }^{1}$ The decision to offer surgery for symptom-free, otherwise healthy people without evidence of ischemia is much more difficult. Do we truly understand which morphologic subtypes predispose patients to increased risk of SCD? Are we justified in offering what essentially 
amounts to a prophylactic procedure, often in settings in which we truly do not know definitively that the patient has a pathologic condition?

If an operation is recommended, what is the best operation, and what do we know about outcomes and risks? Does a "successful" operation (typically either "unroofing" of the anomalous coronary or coronary translocation to associate the ostium with the appropriate sinus along with possible translocation of the pulmonary trunk to the left to reduce the potential for coronary artery compression), confer durable protection? Should patients be allowed to participate in sports? What measures should be taken to prepare for a sudden cardiac event (notification of coaches and teachers, automated external defibrillators at sporting events, etc)? In my experience, the ongoing knowledge of their child's having an AAOCA is a nightmare situation for parents, frequently leading them to demand an operation, even in situations with lack of a clear indication.

In this provocative setting, the community of practitioners in congenital heart disease is experiencing something akin to an "epidemic" of the finding of AAOCA. In response to this reality, the American Association for Thoracic Surgery commissioned a group of us to develop a consensus treatment guidelines document for patients with AAOCA. ${ }^{5}$ As noted previously, the work of this group was challenging in the setting of very limited evidence on which to base recommendations. Our ultimate recommendations were that surgery was only indicated in the setting of AAOLCA with an interarterial course or in the setting of either AAOLCA or AAORCA with documented ischemia. Although we realized that these recommendations were at best quite incomplete, the frustrating truth was that there were limited objective data on the topic of AAOCA, risk stratification, and operative outcomes.

In the context of this background, Jegastheeswaran and colleagues ${ }^{6}$ have made important presentations at successive plenary sessions at the annual meeting of the American Association for Thoracic Surgery in 2018 and 2019 on the subject of AAOCA. Their work represents important analyses of data from the Congenital Heart Surgeons' Society multi-institutional AAOCA registry. The data are derived from a voluntary AAOCA registry contributed to by 45 participating institutions and include anatomic details, screening information, operative procedure specifics, and short-term outcomes. The registry includes 682 patients with AAOCA, 395 of whom have undergone an operation. The analyses of Jegastheeswaran and colleagues ${ }^{6}$ reveal a number of very important observations; unfortunately, however, there may be more questions than answers. These include the following:

1. African Americans may have a disproportionate risk of ischemia. Why?
2. Individuals with AAORCA are less likely to have ischemia, but this variant is not totally benign. In AAORCA with an intramural course, a longer length of intramurality appears to be associated with increased risk. Patients with AAORCA have died while under expectant management.

3. Despite the guidelines noted previously, a large percentage of patients with incidentally found AAORCA (and no evidence of ischemia) are undergoing surgery. This may be explained, at least in part, by the disabling nature of the diagnosis alluded to previously.

4. In patients with either AAOLCA or AAORCA, the longer the intramural segment, the greater the risk. This brings up important questions about accurately measuring intramurality and what length of intramural segment meets clear criteria for an operation.

5. Despite guideline recommendations to the contrary, patients without symptoms or a history of SCD are undergoing operations without preoperative provocative testing. Why is this? Should we not be uniform in approach? How will we unravel the details of indications and outcomes without more complete testing?

6. In patients undergoing surgery, outcomes data are concerning. These operations are not risk free and are not always accomplishing what they were designed to do. Furthermore, the Congenital Heart Surgeons' Society AAOCA registry is composed of patients operated on by congenital heart surgeons; surgeons who should be well versed in the delicate nature of manipulating coronary ostia. It thus may be that these data undervalue the risk of AAOCA surgery in general cardiothoracic practice (as anecdote does suggest). Worrisome findings from current registry analyses include the following:

- Patients with AAOCA are largely being treated with "unroofing" procedures, but if this is associated with manipulation of an associated aortic valve pillar or commissure, there is a significant risk of new, important aortic insufficiency.

- A significant number of patients who undergo operation have evidence of ischemia after surgery, including patients who did not have ischemia before surgery (in this registry, $3 \%$ of patients operated on).

- After surgery, $2 \%$ of patients have new, important left ventricular dysfunction.

- There is a significant need for reoperation after the primary, index surgery, including patients who need remedial surgery for a coronary artery misadventure.

- Symptom-free patients without documented ischemia have died after operations that were designed to protect them.

Although sobering, these data are critical to current practice and must be shared with practitioners, patients, and 
families. We do not know everything that we need to know, but we know a lot more than previously, and it is not all good news. Despite these concerns, the reports underscore the absolute necessity of robust, multi-institutional data repositories for patients with AAOCA. The data elements must be expanded, including standardized, objective reporting methods of the precise anatomic and physiologic details (determining length of intramural segment, provocative testing for ischemia, etc) and terminology consistency. There may be a need for a core imaging center to independently corroborate anatomy. Patients must be followed longitudinally, probably for life. The data must be frequently updated and reanalyzed. We must involve patients and parents in developing quantitative, patientderived outcomes measures, including critically analyzing the psychosocial burden associated with the diagnosis of AAOCA. In the meantime, the field will continue to navigate in the fog as best we are able.

\section{Conflict of Interest Statement}

The authors reported no conflicts of interest.

The Journal policy requires editors and reviewers to disclose conflicts of interest and to decline handling or re- viewing manuscripts for which they may have a conflict of interest. The editors and reviewers of this article have no conflicts of interest.

\section{References}

1. Mery CM, Lawrence SM, Krishnamurthy R, Sexson-Tejtel SK, Carberry KE, McKenzie ED, et al. Anomalous aortic origin of a coronary artery: toward a standardized approach. Semin Thorac Cardiovasc Surg. 2014;26:110-22.

2. Maron BJ, Doerer JJ, Haas TS, Tierney DM, Mueller FO. Sudden deaths in young competitive athletes: analysis of 1866 deaths in the United States, 1980-2006. Circulation. 2009;119:1085-92.

3. Eckart RE, Scoville SL, Campbell CL, Shry EA, Stajduhar KC, Potter RN, et al. Sudden death in young adults: a 25-year review of autopsies in military recruits. Ann Intern Med. 2004;141:829-34.

4. Angelini P, Cheong BY, Lenge De Rosen VV, Lopez A, Uribe C, Masso AH, et al. High-risk cardiovascular conditions in sports-related sudden death: prevalence in 5,169 schoolchildren screened via cardiac magnetic resonance. Tex Heart Inst J. 2018;45:205-13.

5. Brothers JA, Frommelt MA, Jaquiss RDB, Myerburg RJ, Fraser CD Jr, Tweddell JS. Expert consensus guidelines: anomalous aortic origin of a coronary artery. J Thorac Cardiovasc Surg. 2017;153:1440-57.

6. Jegatheeswaran A, Devlin PJ, McCrindle BW, Williams WG, Jacobs ML, Blackstone EH, et al. Features associated with myocardial ischemia in anomalous aortic origin of a coronary artery: a A Congenital Heart Surgeons' Society study. J Thorac Cardiovasc Surg. 2019;158:822-34.e3.

Key Words: anomalous coronary arteries, surgical risk, longitudinal outcomes 